\title{
Superderivations and Symmetric Markov Semigroups
}

\author{
E. Brian Davies ${ }^{1}$ and J. Martin Lindsay ${ }^{2}$ \\ ${ }^{1}$ Department of Mathematics, King's College, London WC2R 2LS, England \\ ${ }^{2}$ Department of Mathematics, University Park, Nottingham NG7 2RD, England
}

Received November 9, 1992

\begin{abstract}
Unbounded superderivations are used to construct non-commutative elliptic operators on semi-finite von Neumann algebras. The method exploits the interplay between dynamical semigroups and Dirichlet forms. The elliptic operators may be viewed as generators of irreversible dynamics for fermion systems with infinite degrees of freedom.
\end{abstract}

\section{Introduction}

A new link between Markov semigroups and superderivations is demonstrated. By establishing a Dirichlet property for a class of superderivations we are able to apply the theory of non-commutative symmetric Markov semigroups ([AH-K, DL]) to the construction of dynamical semigroups on $\mathbb{Z}_{2}$-graded algebras of quantum observables. Such a theory is required for describing the irreversible dynamics of infinite systems of fermionic particles (cf. [D1, D2]). The theory described here is applicable to tracial states, and thus involves an infinite temperature assumption. To deal with non-tracial KMS states requires extensive generalisation of [AH-K] and [DL]. In particular the non-commutative $L^{p}$-spaces of Segal ([Seg]) must be replaced by those of Haagerup ([Haa]). A theory of KMS-symmetric Markov semigroups has recently been developed ([GL1, 2]).

Derivations have long played a part in the construction of dynamical semigroups, stemming from the fact that if a derivation generates an automorphism group, then its square generates a completely positive semigroup which may be expressed as a gaussian average of the automorphisms ([Ev, D]). The possibility of exploiting superderivations arises from a change in point of view. If one considers Markov semigroups acting on the $L^{2}$-space of the algebra with respect to a semifinite trace, and views the generator as a quadratic form, then the positivity and contractivity of the semigroup is reflected in a Dirichlet property for the form, as in the classical theory ([Fuk]). Superderivations now yield Dirichlet forms as obligingly as derivations do, moreover the powerful analytic tool of quadratic forms is well suited to dealing with infinite families of superderivations. 
We first develop the elementary theory of superderivations. In the second section some useful results about Hilbert algebras are collected. Next unbounded superderivations are analysed, and in the final section the techniques developed in ([DL]) are applied to superderivations.

\section{Superderivations on a $\mathbb{Z}_{2}$-Graded Algebra}

Let $(\mathscr{A}, \Gamma)$ be a $\mathbb{Z}_{2}$-graded associative algebra, so that $\Gamma$ is an automorphism of $\mathscr{A}$ which is involutive: $\Gamma^{2}=\mathrm{id}$. Thus $\mathscr{A}$ is a direct sum of $\mathscr{A}_{+}:=\left\{x \in \mathscr{A}: x^{\Gamma}=x\right\}$ and $\mathscr{A}_{-}:=\left\{x \in \mathscr{A}: x^{\Gamma}=-x\right\}$. The elements of $\mathscr{A}_{+}$are called even and those of $\mathscr{A}_{-}$odd. If the algebra has an identity element $e$, then $e$ is even. When the algebra is unital, the $\mathbb{Z}_{2}$-grading is called inner if there is an invertible element $\gamma \in \mathscr{A}$ such that

$$
\gamma^{-1}=\gamma \quad \text { and } \quad x^{\Gamma}=\gamma x \gamma \quad \forall x \in \mathscr{A} .
$$

Note that any implementing $\gamma$ is necessarily even.

A superderivation on $(\mathscr{A}, \Gamma)$ is a linear map $\delta$ on $\mathscr{A}$ satisfying:

$$
\begin{aligned}
\text { (i) } \delta(x y) & =\delta x \cdot y+x^{\Gamma} \delta y \\
\text { (ii) } \delta\left(x^{\Gamma}\right) & =-(\delta x)^{\Gamma} \quad \forall x, y \in \mathscr{A} .
\end{aligned}
$$

Thus $\delta\left(\mathscr{A}_{ \pm}\right) \subset \mathscr{A}_{\mp}$ and, if $\mathscr{A}$ has a unit $e$, then $\delta e=0$. Whereas (i) appears to generalise the notion of a derivation to take into account non-trivial gradings of the algebra $\left(\mathscr{A}_{-} \neq\{0\}\right)$, (ii) implies that the only superderivation on a trivially graded algebra $(\Gamma=\mathrm{id})$ is the zero map. A superderivation $\delta$ is not usually a derivation itself - necessary and sufficient conditions on $\delta$ being

$$
x \cdot \delta y=0 \quad \forall x \in \mathscr{A}_{-}, \quad y \in \mathscr{A}
$$

- however its square $\delta^{2}$ is always a derivation. We wish to know when a superderivation is inner - that is when there is $a \in \mathscr{A}$ such that

$$
\delta x=a x-x^{\Gamma} a \quad \forall x \in \mathscr{A} .
$$

First we show that $a$ may be assumed to be odd.

Lemma 1.1. Let $\delta$ be an inner superderivation on a unital $\mathbb{Z}_{2}$-graded algebra $(\mathscr{A}, \Gamma)$, then $\delta=\delta_{a}$ where $a \in \mathscr{A}$ is odd.

Proof. Since $\delta$ is inner, $\delta=\delta_{c}$ for some $c \in \mathscr{A}$. By (1.1) $c$ must satisfy

$$
c x-x^{\Gamma} c=-\left(\delta_{c}\left(x^{\Gamma}\right)\right)^{\Gamma}=-\left(c x^{\Gamma}-x c\right)^{\Gamma}=-c^{\Gamma} x+x^{\Gamma} c^{\Gamma} \quad \forall x \in \mathscr{A} .
$$

In other words $\delta_{\left(c+c^{r}\right)} \equiv 0$. Thus if $a=\frac{1}{2}\left(c-c^{\Gamma}\right)$, then $\delta=\delta_{a}$ and $a$ is odd.

Lemma 1.2. Let $(\mathscr{A}, \Gamma)$ be a unital $\mathbb{Z}_{2}$-graded algebra. If $\Gamma$ is inner then every superderivation on $(\mathscr{A}, \Gamma)$ is inner.

Proof. Let $\delta$ be a superderivation and suppose that $\gamma \in \mathscr{A}$ implements the grading involution, then

$$
\delta \gamma \cdot \gamma=\gamma \cdot(\delta \gamma)^{\Gamma}=-\gamma \delta \gamma
$$


Therefore, for $x \in \mathscr{A}$,

$$
\begin{aligned}
\delta x=-\left(\delta\left(x^{\Gamma}\right)\right)^{\Gamma} & =-\gamma \delta(\gamma x \gamma) \gamma \\
& =-\gamma\left\{\delta \gamma \cdot x \gamma+\gamma^{\Gamma} \delta(x \gamma)\right\} \gamma \\
& =-\gamma \delta \gamma \cdot x-\left(\delta x \cdot \gamma+x^{\Gamma} \delta \gamma\right) \gamma \\
& =\delta \gamma \cdot \gamma x-\delta x-x^{\Gamma} \delta \gamma \cdot \gamma .
\end{aligned}
$$

In other words, $\delta=\delta_{a}$ where $a=\frac{1}{2} \delta \gamma \cdot \gamma$. Note that $a$ is odd since it is the product of an even element and an odd one.

\section{Hilbert Algebras}

Let $\mathscr{A}$ be a fixed von Neumann algebra with faithful, normal semi-finite trace $\tau$. For $p \in[1, \infty]$, let $L^{p}$ denote the corresponding non-commutative $L^{p}$-space in the sense of I.E. Segal ([Seg, Kun, Yea]), and let $\tilde{\mathscr{A}}$ denote the associated topological *-algebra of $\tau$-measurable operators $([\mathrm{Nel}])$. Thus $\tilde{\mathscr{A}}$ consists of closed, densely defined operators, and the algebraic operations are "strong sense":

$$
(x, y) \mapsto \overline{x+y}, \overline{x-y}, \overline{x y} ; \quad(\lambda, x) \mapsto \overline{\lambda x} \quad x, y \in \tilde{\mathscr{A}}, \lambda \in \mathbb{C} .
$$

All sums, products etc. of elements from $\tilde{\mathscr{A}}$ are to be understood in this strong sense. The topology of $\tilde{\mathscr{A}}$ is given by convergence in measure ([Sti, Nel]). $\tilde{\mathscr{A}}$ contains all the $L^{p}$-spaces and, most conveniently, the Hilbert space on which the elements of $\tilde{\mathscr{A}}$ act is $L^{2}$ itself - the action being strong sense (left) multiplication. $L^{\infty}$ is then a faithful normal representation of $\mathscr{A}$, and $L^{\infty} \cap L^{2}$ is a Hilbert algebra ([Dix]). The inner product $(x, y) \mapsto \tau\left(x^{*} y\right)$ on $L^{2}$ extends to a sesquilinear pairing $\langle$,$\rangle on L^{p} \times L^{p^{\prime}}$, for each $p \in[1, \infty]$. These satisfy

$$
\left\langle x^{*}, y^{*}\right\rangle=\langle y, x\rangle ; \quad\langle a x, y\rangle=\left\langle x, a^{*} y\right\rangle ; \quad\langle x a, y\rangle=\left\langle x, y a^{*}\right\rangle
$$

whenever the expressions are defined $-a x \in L^{p}, y \in L^{p^{\prime}}$ for some $p$, etc. The following result is a consequence of this representation.

Lemma 2.1. If $a \in \tilde{\mathscr{A}}$ satisfies

$$
a x=0 \quad \forall x \in \mathscr{D},
$$

where $\mathscr{D}$ is a dense subspace of $L^{2}$, then $a=0$.

We mention next a density result which will be useful in our analysis of unbounded superderivations.

Proposition 2.2. Let $\mathscr{D}$ be a dense subspace of $L^{2}$ contained in $L^{2} \cap L^{\infty}$. Then $\mathscr{D} \cdot \mathscr{D}:=$ linear span $\{x y: x, y \in \mathscr{D}\}$ is dense in $L^{1} \cap L^{2}$ for the norm $x \mapsto\|x\|_{1}+\|x\|_{2}$.

Proof. Let $a \in L^{\infty}+L^{2}$ satisfy

$$
\langle a, x\rangle=0 \quad \forall x \in \mathscr{D} \cdot \mathscr{D} .
$$

Then, for each $v \in \mathscr{D}$,

$$
\begin{aligned}
0=\langle a, u v\rangle & =\tau\left(a^{*} u v\right) \\
& =\tau\left(v a^{*} u\right)=\left\langle a v^{*}, u\right\rangle \quad \forall u \in \mathscr{D} .
\end{aligned}
$$


Since $a v^{*} \in\left(L^{2} \cap L^{\infty}\right) \cdot\left(L^{\infty}+L^{2}\right) \subset L^{2}$, and $\mathscr{D}$ is $L^{2}$-dense, $a v^{*}=0$. By Lemma $2.1 a=0$ and, since $L^{\infty}+L^{2}=\left(L^{1} \cap L^{2}\right)^{*}$, the assertion follows from the HahnBanach theorem.

Proposition 2.3. Let $a \in L^{\infty}+L^{2}$. Then, for sufficiently large $N, a e^{|a|}[N, \infty) \in L^{2}$, where $e^{|a|}(\cdot)$ is the spectral measure of $|a|$.

Proof. Since $|a|^{2}=a^{*} a \in\left(L^{\infty}+L^{2}\right) \cdot\left(L^{\infty}+L^{2}\right)=L^{\infty}+L^{1}$, we may write $|a|^{2}=b+c$, where $b \in L^{1}$ and $c \in L^{\infty}$. Choose an integer $N$ such that

$$
\text { (i) } N^{2} \geqq 2\|c\|_{\infty}+1 ; \text { (ii) } \tau\left(e^{|a|^{2}}[M, M+1)\right)<\infty \text { for } M \geqq N^{2} \text {. }
$$

(ii) is ensured for some $N$ since $|a| \in \tilde{\mathscr{A}}$, but in fact (i) implies (ii). By Hölder's inequality

$$
\begin{aligned}
\sum_{k=N^{2}}^{M} k \tau\left(e^{|a|^{2}}[k, k+1)\right) & \leqq \tau\left(|a|^{2} e^{|a|^{2}}\left[N^{2}, M+1\right)\right) \\
& \leqq\|b\|_{1}+\|c\|_{\infty} \tau\left(e^{|a|^{2}}\left[N^{2}, M+1\right)\right)
\end{aligned}
$$

so, by (ii) and (i),

$$
\begin{aligned}
\frac{1}{2} \tau\left(|a|^{2} e^{|a|^{2}}\left[N^{2}, M+1\right)\right) & \leqq \sum_{k=N^{2}}^{M}\left(\frac{k+1}{2}\right) \tau\left(e^{|a|^{2}}[k, k+1)\right) \\
& \leqq \sum_{k=N^{2}}^{M}\left\{k-\|c\|_{\infty}\right\} \tau\left(e^{|a|^{2}}[k, k+1)\right) \leqq\|b\|_{1} .
\end{aligned}
$$

By the normality of $\tau$ if we let $M \mapsto \infty$ we obtain

$$
\left\|a e^{|a|}[N, \infty)\right\|_{2}^{2} \leqq 2\|b\|_{1}<\infty .
$$

Thus elements of $L^{\infty}+L^{2}$ may be expressed as a sum of an $L^{\infty}$-part and an $L^{2}$-part in the most straightforward fashion. [DL] includes a summary of $L^{p}$-theory, and we recommend $[\mathrm{Nel}]$ for a nice full account.

\section{Superderivations on $\mathbb{Z}_{2}$-Graded Hilbert Algebras}

From now on $\mathscr{A}$ will be a fixed von Neumann algebra with $\mathbb{Z}_{2}$-grading involution $\Gamma$ and faithful, normal, semi-finite trace $\tau$. The trace is assumed to be $\Gamma$-invariant, and the grading involution is assumed to be a ${ }^{*}$-automorphism: $\left(x^{*}\right)^{\Gamma}=\left(x^{\Gamma}\right)^{*}$. Involutions, which we continue to denote by $x \mapsto x^{\Gamma}$, are induced on each $L^{p}$ where they satisfy

$$
\left\|x^{\Gamma}\right\|_{L^{p}}=\|x\|_{L^{p}} ; \quad\left\langle x^{\Gamma}, y^{\Gamma}\right\rangle=\langle x, y\rangle
$$

for $p \in[1, \infty], x \in L^{p}$ and $y \in L^{p^{\prime}}$.

We are primarily interested in unbounded superderivations on $\mathbb{Z}_{2}$-graded Hilbert algebras. These are pairs $(\delta, \mathscr{D})$, where $\mathscr{D}$ is a $\Gamma$-invariant $*$-subalgebra of the full Hilbert algebra $L^{2} \cap L^{\infty}$, which is dense in $L^{2}$, and $\delta: \mathscr{D} \rightarrow L^{2}$ is a linear map satisfying the superderivation properties $(1.1) .(\delta, \mathscr{D})$ is a ${ }^{*}$-superderivation if it satisfies the further property

$$
\delta x^{*}=\left((\delta x)^{\Gamma}\right)^{*} \quad \forall x \in \mathscr{D} .
$$


Notice that if $\delta$ is a superderivation, then so is

$$
\delta^{\prime}: x \mapsto\left(\left(\delta x^{*}\right)^{\Gamma}\right)^{*},
$$

thus $\delta$ is a ${ }^{*}$-superderivation precisely when $\delta$ and $\delta^{\prime}$ coincide. Moreover every superderivation $(\delta, \mathscr{D})$ may be written $\delta=\delta_{1}+\mathrm{i} \delta_{2}$, where $\left(\delta_{1}, \mathscr{D}\right)$ and $\left(\delta_{2}, \mathscr{D}\right)$ are

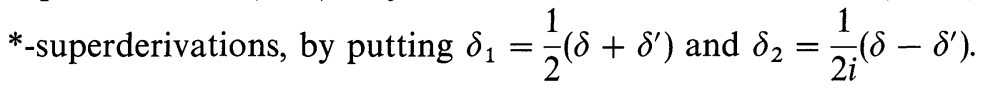

Definition 3.1. A superderivation $(\delta, \mathscr{D})$ on a $\mathbb{Z}_{2}$-graded Hilbert algebra is inner if there is $a \in L^{\infty}+L^{2}$ such that

$$
\delta=\delta_{a}: x \mapsto a x-x^{\Gamma} a .
$$

By the argument of Lemma 1.1, a may always be chosen to be odd. It is easily checked that

$$
\delta_{a}^{\prime}=\delta_{a^{*}} ;
$$

therefore inner ${ }^{*}$-superderivations are all of the form $\delta_{a}$ where $a^{*}=a=$ $-a^{\Gamma} \in L^{\infty}+L^{2}$.

Definition 3.2. A superderivation on a $\mathbb{Z}_{2}$-graded Hilbert algebra is smooth if, for some element $b$ of $L^{\infty}+L^{2}$,

$$
\langle 1, \delta u\rangle=\langle b, u\rangle \quad \forall u \in \mathscr{D} \cdot \mathscr{D} .
$$

By Proposition 2.2 when such a $b$ exists, it is uniquely determined and, by an abuse of notation we shall denote it by $\delta^{*} 1$. We first show that $\delta^{*} 1$ is necessarily odd.

Lemma 3.3. Let $(\delta, \mathscr{D})$ be a superderivation on a $\mathbb{Z}_{2}$-graded Hilbert algebra. If $\delta$ is smooth then $\delta^{*} 1$ is odd. Moreover, if $\delta$ is a ${ }^{*}$-superderivation, then $\delta^{*} 1$ is self-adjoint also.

Proof. For $x \in \mathscr{D} \cdot \mathscr{D}$,

$$
\begin{aligned}
\left\langle b^{\Gamma}, x\right\rangle=\left\langle b, x^{\Gamma}\right\rangle & =\left\langle 1, \delta\left(x^{\Gamma}\right)\right\rangle \\
& =-\left\langle 1,(\delta x)^{\Gamma}\right\rangle=-\langle b, x\rangle .
\end{aligned}
$$

Therefore $b$ is odd by Proposition 2.2. If $\delta$ is a *-superderivation, then

$$
\begin{aligned}
\langle b, x\rangle & =\left\langle 1, \delta\left(x^{* *}\right)\right\rangle \\
& =\left\langle 1,\left(\left(\delta\left(x^{*}\right)\right)^{\Gamma}\right)^{*}\right\rangle \\
& =\left\langle\delta\left(x^{*}\right), 1\right\rangle \\
& =\left\langle x^{*}, b\right\rangle=\left\langle b^{*}, x\right\rangle \quad \forall x \in \mathscr{D} \cdot \mathscr{D},
\end{aligned}
$$

so Proposition 2.2 implies that $b$ is self-adjoint.

The next result is in contrast to the situation with derivations. Inner derivations are clearly smooth in the sense of Definition $3.2(b=0)$, however commutative algebras such as $L^{2} \cap L^{\infty}(\mathbb{R}$, Leb) carry plenty of smooth derivations (vector fields with bounded derivative). 
Theorem 3.4. A superderivation $(\delta, \mathscr{D})$ on a $\mathbb{Z}_{2}$-graded Hilbert algebra is inner if and only if it is smooth. In this case

$$
\delta=\delta_{a} \quad \text { and } \quad \delta^{*} \supset L_{\delta^{*} 1}-\delta^{\prime},
$$

where $a=\frac{1}{2}\left(\delta^{*} 1\right)^{*}, \delta^{\prime}$ is given by (3.1) and $L_{x}$ denotes left multiplication by $x$. In particular $\delta$ is closable. Moreover a is odd and, if $(\delta, \mathscr{D})$ is $a^{*}$-superderivation then a is also self-adjoint.

Proof. First assume that $(\delta, \mathscr{D})$ is smooth and let $b=\delta^{*} 1 \in L^{2}+L^{\infty}$. Then, for $x, y \in \mathscr{D}$,

$$
\begin{aligned}
\langle x, \delta y\rangle & =\left\langle 1, \delta y \cdot x^{*}\right\rangle \\
& =\left\langle 1, \delta\left(y x^{*}\right)-y^{\Gamma} \delta x^{*}\right\rangle \\
& =\left\langle b, y x^{*}\right\rangle-\left\langle\delta^{\prime} x, y\right\rangle \\
& =\left\langle b x-\delta^{\prime} x, y\right\rangle .
\end{aligned}
$$

Thus $\delta^{*} \supset L_{b}-\delta^{\prime}$, whose $L^{2}$-domain is $\mathscr{D}$ which is $L^{2}$-dense. In particular $\delta$ is closable. On the other hand, for $x, y \in \mathscr{D}$,

$$
\begin{aligned}
\langle x, \delta y\rangle & =\left\langle 1, x^{*} \cdot \delta y\right\rangle \\
& =\left\langle 1, \delta\left(\left(x^{*}\right)^{\Gamma} y\right)-\delta\left(\left(x^{*}\right)^{\Gamma}\right) \cdot y\right\rangle \\
& =\left\langle b,\left(x^{*}\right)^{\Gamma} y\right\rangle+\left\langle\delta^{\prime} x, y\right\rangle \\
& =\left\langle x^{\Gamma} b+\delta^{\prime} x, y\right\rangle .
\end{aligned}
$$

Subtracting this from (3.3) gives

$$
\delta^{\prime} x=\frac{1}{2}\left(b x-x^{\Gamma} b\right) .
$$

Thus $\delta^{\prime}$ is inner and so, by (3.2) $\delta=\delta_{a}$ where $a=\frac{1}{2}\left(\delta^{*} 1\right)^{*} \in L^{\infty}+L^{2}$.

On the other hand, if $(\delta, \mathscr{D})$ is inner, then $\delta=\delta_{a}$, where $a=-a^{\Gamma} \in L^{\infty}+L^{2}$, so that

$$
\begin{aligned}
\langle 1, \delta x\rangle & =\tau\left(a x-x^{\Gamma} a\right) \\
& =\tau\left(a x-a^{\Gamma} x\right) \\
& =\left\langle 2 a^{*}, x\right\rangle \quad \forall x \in \mathscr{D} \cdot \mathscr{D} .
\end{aligned}
$$

In other words, $(\delta, \mathscr{D})$ is smooth. The rest of the result therefore follows from Lemma 3.3.

As a corollary we may characterise the $L^{2}$-bounded superderivations.

Theorem 3.5. $A$ superderivation $(\delta, \mathscr{D})$ on a $\mathbb{Z}_{2}$-graded Hilbert algebra is $L^{2}$-norm bounded if and only if $\delta=\delta_{a}$, where $a \in L^{\infty}$. In this case $a$ is odd and, if $(\delta, \mathscr{D})$ is $a{ }^{*}$-superderivation, then $a$ is self-adjoint too. 
Proof. We first show that $(\delta, \mathscr{D})$ extends to a superderivation on $L^{2} \cap L^{\infty}$. Let $\bar{\delta}$ be the continuous extension of $\delta$ to $L^{2}$. Then for $x, y, z \in L^{2} \cap L^{\infty}$,

$$
\begin{aligned}
\langle z, \bar{\delta}(x y)\rangle & =\left\langle x^{*} \delta^{*} z, y\right\rangle \\
& =\lim _{n \rightarrow \infty}\left\langle x^{*} \delta^{*} z, y_{n}\right\rangle \\
& =\lim _{n \rightarrow \infty} \lim _{m \rightarrow \infty}\left\langle x_{m}^{*} \delta^{*} z, y_{n}\right\rangle \\
& =\lim _{n \rightarrow \infty} \lim _{m \rightarrow \infty}\left\langle z, \delta\left(x_{m} y_{n}\right)\right\rangle,
\end{aligned}
$$

where $\left(x_{m}\right)$ and $\left(y_{n}\right)$ are sequences in $\mathscr{D}$ which converge to $x$ and $y$ respectively, in the $L^{2}$-norm. Applying the super-Leibnitz property and unravelling the limits we see that $\left(\bar{\delta}, L^{2} \cap L^{\infty}\right)$ is a superderivation. We may therefore assume without loss that $\mathscr{D}=L^{2} \cap L^{\infty}$.

The linear functional

$$
\varphi: u \in \mathscr{D} \cdot \mathscr{D} \mapsto\langle 1, \delta u\rangle
$$

is $L^{1}$-densely defined since $\mathscr{D} \cdot \mathscr{D}=L^{1} \cap L^{\infty}$ is dense in $L^{1}$. Moreover, by means of the polar decomposition $u=v|u|$, each $u \in \mathscr{D} \cdot \mathscr{D}$ may be written as a product $u=x y$, where $x=v|u|^{\frac{1}{2}} \in \mathscr{D}, y=|u|^{\frac{1}{2}} \in \mathscr{D}$ and $\|u\|_{L^{1}}=\|x\|_{L^{2}}^{2}=\|y\|_{L^{2}}^{2}$. Then

$$
\begin{aligned}
|\varphi(u)| & =\left|\left\langle 1, \delta y \cdot x+y^{\Gamma} \cdot \delta x\right\rangle\right| \\
& =\left|\left\langle x^{*}, \delta y\right\rangle+\left\langle\left(y^{*}\right)^{\Gamma}, \delta x\right\rangle\right| \\
& \leqq\|\delta\|\|x\|_{L^{2}}\|y\|_{L^{2}}=\|\delta\|\|u\|_{L^{1}},
\end{aligned}
$$

where $\|\delta\|$ is the $L^{2}$-operator norm of $\delta$, so $\varphi$ has unique continuous extension to $L^{1}$. The duality theory of non-commutative $L^{p}$-spaces ensures the existence of $b \in L^{\infty}$ such that $\langle 1, \delta u\rangle=\langle b, u\rangle \forall u \in \mathscr{D} \cdot \mathscr{D}$ - in other words $\delta$ is smooth. By Theorem $3.4 \delta=\delta_{a}$, where $a=\frac{1}{2}\left(\delta^{*} 1\right)^{*} \in L^{\infty}+L^{2}$, moreover the relation $\delta^{*} \supset L_{\left(\delta^{*} 1\right)}-\delta^{\prime}$ implies that

$$
L_{\left(\delta^{*} 1\right)}=\delta^{*}+\overline{\delta^{\prime}},
$$

since $\delta^{*}$ and $\delta^{\prime}$ are $L^{2}$-bounded and $L^{2} \cap L^{\infty}$ is a core for $L_{\left(\delta^{*}\right)}$. But a (left) multiplication operator $L_{b}$ can only be bounded if $b \in L^{\infty}$ - this follows from the duality relation $\left(L^{1}\right)^{*}=L^{\infty}$. Hence $\delta^{*} 1 \in L^{\infty}$ and the result follows.

\section{Symmetric Markov Semigroups}

We recall the basic definition from ([DL]). A $c_{0}$-semigroup $\left(P_{t}\right)$ on $L^{2}$ is called a Markov semigroup if

(i) $P_{t} \geqq 0$ whenever $x \geqq 0$;

(ii) $P_{t} x \leqq 1$ whenever $x \leqq 1$.

In other words, each $P_{t}$ is positivity preserving and $L^{\infty}$-contractive on $\left(L^{2} \cap L^{\infty}\right)_{\text {s.a. }}$. A Markov semigroup is symmetric if each $P_{t}$ is a self-adjoint operator on $L^{2}$. Symmetric Markov semigroups extend to semigroups $\left(P_{t}^{(p)}\right)$ on $L^{p}$, which are strongly continuous for $1 \leqq p<\infty$, and pointwise weak ${ }^{*}$-continuous for $p=\infty$. 
The extensions are consistent: if $x \in L^{p} \cap L^{q}$ then $P_{t}^{(p)} x=P_{t}^{(q)} x$, therefore we may drop the superscripts. A symmetric Markov semigroup is called conservative if

(ii)' $P_{t} 1=1$ for $t \geqq 0$,

and completely positive if

$$
\sum_{i, j=1}^{n} y_{i}^{*} P_{t}\left(x_{i}^{*} x_{j}\right) y_{j} \geqq 0 \text { for } t \geqq 0, n \in \mathbb{N}, x_{1}, y_{1}, \ldots, x_{n}, y_{n} \in L^{\infty} .
$$

On the otherhand if $\left(P_{t}\right)$ is a pointwise weak *-continuous semigroup of normal maps on $\mathscr{A}$ satisfying (i), (ii) and tracial symmetry:

$$
\tau\left(x \cdot P_{t} y\right)=\tau\left(P_{t} x \cdot y\right) \quad \forall x, y \in\left(L^{1} \cap L^{\infty}\right)(\mathscr{A}, \tau)
$$

then $\left(P_{t}\right)$ determines a symmetric Markov semigroup on $L^{2}$. Thus we have equivalent algebra and Hilbert space pictures for (tracially) symmetric Markov semigroups.

We shall see that inner *-superderivations lead to symmetric Markov semigroups in a similar way to inner *-derivations ([DL]). We begin with the bounded case.

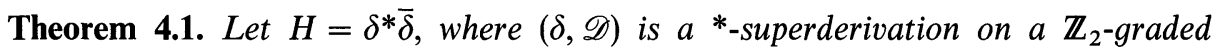
Hilbert algebra. If $\delta$ is $L^{2}$-norm bounded then $\left(e^{-t H}\right)$ is a completely positive, conservative, symmetric Markov semigroup.

Proof. By Theorem $3.5 \delta=\delta_{a}$, where $a \in L^{\infty}$ is odd and self-adjoint. Therefore $\delta^{*}: x \in L^{2} \mapsto a x+x^{\Gamma} a$. For $p \in[1, \infty]$ let $\left(P_{t}^{(p)}=e^{-t H^{(t)}}\right)$, where

$$
H^{(p)}: x \in L^{p} \mapsto a^{2} x+x a^{2}-2 a x^{\Gamma} a .
$$

A direct computation verifies that $H^{(2)}=H$, that is $\delta^{*} \bar{\delta}$. $\left\{\left(P_{t}^{(p)}\right): p \in[1, \infty]\right\}$ form a consistent family of uniformly continuous semigroups. Since $H$ is manifestly self-adjoint and also real: $H\left(x^{*}\right)=(H x)^{*}, \tau\left(x \cdot H^{(\infty)} y\right)=\tau\left(x \cdot H^{(2)} y\right)=\tau\left(H^{(1)} x \cdot y\right)$ $\forall x, y \in L^{1} \cap L^{\infty}$ so that $\left(P_{t}^{(\infty)}\right)$ is $\tau$-symmetric and is the dual semigroup to $\left(P_{t}^{(1)}\right)$ in particular it consists of normal maps. Now $H^{(\infty)}=L+M$, where

$$
e^{-t L}: x \mapsto e^{-t a^{2}} x e^{-t a^{2}} \text { and } e^{-t M}: x \mapsto \sum_{n \geqq 0} \frac{(2 t)^{n}}{n !} a^{n} x^{\Gamma} a^{n} .
$$

Since $\Gamma$ is an automorphism, the Trotter product formula implies that $P_{t}^{(\infty)}$ is completely positive for each $t$. Finally $P_{t}^{(\infty)} 1=1$ for each $t$ since $H^{(\infty)} 1=0$ and so each $P_{t}^{(\infty)}$ is conservative and Markov.

Notice that the above Markov semigroup also preserves parity: $P_{t}\left(x^{\Gamma}\right)=$ $\left(P_{t} x\right)^{T}$. There is a very nice stochastic description of Markov semigroups of the above form. Namely the semigroup may be viewed as a quantum average of a stochastic flow:

$$
e^{-t H} x=\mathbb{E}\left[u_{t} x u_{t}^{*}\right], \quad t \geqq 0,
$$

where $u_{t}$ satisfies a stochastic differential equation involving Fermionic Brownian motion (see $[\mathrm{ApH}]$, Theorem 7.1(b)). In fact quantum stochastics has an alternative, more algebraic view of such semigroups. Let $\left(A^{*}, \Lambda, A\right)$ be the creation, preservation, annihilation process triple of quantum stochastic calculus $([\mathrm{HuP}$, Par]). Then, for any element $b$ of $L^{\infty}$, there is a family of unital *-homomorphisms 
$\left(j_{t}\right)_{t \geq 0}$ from $L^{\infty}$ into a larger algebra satisfying the quantum stochastic differential equation

$$
d j_{t}=j_{t} \circ(\Gamma-\mathrm{id}) d \Lambda+j_{t} \circ \delta d A^{*}+j_{t} \circ \delta^{\dagger} d A+j_{t} \circ \Delta d t,
$$

where $\delta=\delta_{b}, \delta^{\dagger}: x \mapsto\left(\delta x^{*}\right)^{*}$, and

$$
\left(\mathbb{E} \circ j_{t}\right)_{t \geqq 0}
$$

is a completely positive, conservative Markov semigroup with generator

$$
\Delta: x \mapsto b^{*} b x-2 b^{*} x^{\Gamma} b+x b^{*} b,
$$

([Ev, M; Par]). This semigroup will not be $(\tau-)$ symmetric unless

$$
b^{\Gamma} x\left(b^{\Gamma}\right)^{*}=b^{*} x b \quad \forall x \in L^{\infty} .
$$

To go beyond the bounded case we exploit Dirichlet forms. Define $\operatorname{Lip}_{0}$ to be the class of functions $\varphi: \mathbb{R} \rightarrow \mathbb{R}$ such that

$$
\varphi(0)=0 \quad \text { and }|\varphi(s)-\varphi(t)| \leqq|s-t| \quad \forall s, t \in \mathbb{R} .
$$

Proposition 4.2. Let $(\delta, \mathscr{D})$ be an $L^{2}$-norm bounded ${ }^{*}$-superderivation. Then the following relations hold for $x \in L^{2}$ :

$$
\begin{aligned}
\left\|\bar{\delta} x^{*}\right\|_{L^{2}} & =\|\bar{\delta} x\|_{L^{2}}, \\
\|\bar{\delta}|x|\|_{L^{2}} & \leqq \sqrt{2}\|\bar{\delta} x\|_{L^{2}}, \\
\|\bar{\delta} \varphi(x)\|_{L^{2}} & \leqq\|\bar{\delta} x\|_{L^{2}} \quad\left(x=x^{*}, \varphi \in \operatorname{Lip}_{0}\right) .
\end{aligned}
$$

Proof. Since $\left(e^{-t \delta^{*} \bar{\delta}}\right)$ is a symmetric Markov semigroup, and is 2-positive, the bounds (4.1) hold on $L^{2} \cap L^{\infty}$ by Propositions 2.12, 3.3 and 3.4 of [DL]. The full result follows from the density of $L^{2} \cap L^{\infty}$ in $L^{2}$ and the $L^{2}$-continuity of the absolute value map ([ArY]) and Lipschitz maps ([AH-K $]$ ) - see Propositions 1.2 and 2.5 of [DL].

We now extend these results to unbounded inner *-superderivations.

Proposition 4.3. Let $(\delta, \mathscr{D})$ be an inner *-superderivation (Definition 3.1) with $\mathscr{D}=L^{2} \cap L^{\infty}$. Then the non-negative quadratic form $Q=\left(\|\delta \cdot\|_{L^{2}}\right)^{2}$ is closable and completely Dirichlet - that is, for each $n \in \mathbb{N}$ and $X \in\left(L^{2} \cap L^{\infty}\right)\left(\mathscr{A} \otimes M_{n}(\mathbb{C})\right)$,

$$
\begin{array}{cc}
Q^{(n)} X^{*}=Q^{(n)} X ; & Q^{(n)}|X| \leqq 2 Q^{(n)} X \\
Q^{(n)} \varphi(X) \leqq Q^{(n)} X & \left(X=X^{*}, \varphi \in \operatorname{Lip}_{0}\right),
\end{array}
$$

where $Q^{(n)}$ is the quadratic form on $L^{2} \otimes\left(M_{n}(\mathbb{C})\right.$, tr) given by

$$
X=\left\{x_{i j}\right\} \mapsto \sum_{i, j=1}^{n} Q x_{i j} .
$$

Proof. The closability of $Q$ is equivalent to the closability of $\delta$ which follows from Theorem 3.4. If $\delta=\delta_{a}$, where $a=a^{*}=-a^{\Gamma} \in L^{\infty}+L^{2}$, let $a_{k}=f_{k}(a)$, where

$$
f_{k}(t)=\left\{\begin{aligned}
-k & \text { if } t<-k \\
t & \text { if }|t| \leqq k \\
k & \text { if } t>k, \quad k=1,2, \ldots
\end{aligned}\right.
$$


Then the bounded, self-adjoint element $a_{k}$ is also odd, since $f_{k}$ is an odd function. Now define $Q_{k}^{(n)}$ on $\left(L^{2} \cap L^{\infty}\right)\left(\mathscr{A} \otimes M_{n}(\mathbb{C})\right)$ by $Q_{k}^{(n)} X=\left\|A_{k} X-X^{\Gamma} A_{k}\right\|_{L^{2}}^{2}$ $=\left\|\bar{\delta}_{A_{k}} X\right\|_{L^{2}}^{2}$, where $A_{k}=\operatorname{diag}\left(a_{k}, \ldots, a_{k}\right), \quad\left\{x_{i j}\right\}^{\Gamma}=\left\{x_{i j}^{\Gamma}\right\}$ and the trace on $\left(\mathscr{A} \otimes M_{n}(\mathbb{C})\right)$ is given by $\tau\left\{x_{i j}\right\}=\sum_{i=1}^{n} \tau x_{i i}$. Since $A_{k} \in L^{\infty}$, Proposition 4.2 implies the completely Dirichlet relations (4.2) for $Q_{k}^{(n)}$. By Proposition $2.3\left(A-A_{k}\right) \in L^{2}$ for sufficiently large $k$, moreover

$$
\left\|A-A_{k}\right\|_{L^{2}}^{2}=\int_{k}^{\infty}(\lambda-k)^{2} d \tau \circ e^{A}(\lambda) \rightarrow 0 \quad \text { as } k \rightarrow \infty,
$$

so $\delta_{A_{k}} X \rightarrow \delta_{A} X$ for each $X$ in $\left(L^{2} \cap L^{\infty}\right)\left(\mathscr{A} \otimes M_{n}(\mathbb{C})\right)$. Therefore $Q^{(n)} X=$ $\lim _{k \rightarrow \infty} Q_{k}^{(n)} X$, and the result follows.

Since the closure of a (completely) Lipschitz form is a (completely) Lipschitz form ([DL] Proposition 2.6), the relations (4.1) and (4.2) remain valid on the domains of the closures of $\delta$ and $Q^{(n)}$ respectively. The following theorem provides a precise technical condition under which one can assert that the expression

$$
H x=\sum_{n \geqq 1}\left(a_{n}^{2} x-2 a_{n} x^{\Gamma} a_{n}+x a_{n}^{2}\right)
$$

determines a non-negative self-adjoint operator $H$ on $L^{2}$ which generates a symmetric Markov semigroup.

Theorem 4.4. Let $\left(\delta_{k}, \mathscr{D}_{k}\right)$ be a sequence of closable *-superderivations on a $\mathbb{Z}_{2}$-graded Hilbert algebra, let

$$
Q: x \in L^{2} \mapsto \begin{cases}\sum_{k \geqq 1}\left(\left\|\bar{\delta}_{k} x\right\|_{L^{2}}\right)^{2} & \text { if } x \in \bigcap_{k \geqq 1} \operatorname{Dom}\left(\bar{\delta}_{k}\right) \\ \infty & \text { otherwise, }\end{cases}
$$

and suppose that $\mathscr{Q}:=\left\{x \in L^{2}: Q x<\infty\right\}$ is $L^{2}$-dense. Then the quadratic form $(Q, 2)$ is closed. If each $\left(\delta_{k}, \mathscr{D}_{k}\right)$ is smooth then $(Q, \mathscr{2})$ is completely Dirichlet. Consequently the associated non-negative operator $H$ generates a completely positive symmetric Markov semigroup.

Proof. $Q$, being a sum of lower semi-continuous functions, is lower semi-continuous and so the quadratic form $(Q, 2)$ is closed. For each $n \in \mathbb{N}$ define

by

$$
Q^{(n)}: \bigcap_{k \geqq 1} \operatorname{Dom}\left(\bar{\delta}_{k}\right) \otimes M_{n}(\mathbb{C}) \rightarrow[0, \infty]
$$

$$
Q^{(n)}\left\{x_{i j}\right\}=\sum_{i, j, k}\left\|\bar{\delta}_{k} x_{i j}\right\|_{L^{2}}^{2},
$$

and write $\mathscr{Q}^{(n)}$ for its natural domain - the set of $X=\left\{x_{i j}\right\}$ for which (4.4) is finite. Then, by Proposition 4.3 and [DL] Proposition 6.4, $\left(Q^{(n)}, \mathscr{Q}^{(n)}\right)$ is a closed Dirichlet form. An appeal to [DL] Theorem 3.3 therefore completes the proof.

Thus, if $a_{k}=a_{k}^{*}=-a_{k}^{\Gamma} \in L^{2}+L^{\infty}$ for $k=1,2, \ldots$, then (4.3) is a well-defined Markov generator provided only that

$$
\sum_{n \geqq 1} \tau\left\{x^{2} a_{n}^{2}+x a_{n}\left(x a_{n}\right)^{\Gamma}\right\}<\infty
$$

for a dense set of $x$ in $L_{\text {s.a. }}^{2}$. 
Formal generators are sometimes given by integrals rather than sums. Our final result shows how these may also be rigorously defined by means of non-commutative Dirichlet forms.

Theorem 4.5. Suppose that the von Neumann algebra $\mathscr{A}$ has separable pre-dual, and let $M$ be a $\sigma$-finite measure space. Define

$$
\begin{aligned}
Q_{b} x & =\left\|\bar{\delta}_{b(\cdot)} x\right\|_{L^{2}\left(M ; L^{2}(\mathscr{A})\right)}^{2}=\int_{M}\left(\left\|\bar{\delta}_{b(m)} x\right\|_{L^{2}(\mathscr{A})}\right)^{2} d m, \\
\mathscr{Q}_{b} & =\left\{x \in L^{2}(\mathscr{A}): Q_{b} x<\infty\right\},
\end{aligned}
$$

where b: $M \rightarrow\left(L^{\infty}+L^{2}\right)(\mathscr{A})$ takes odd, self-adjoint values, and satisfies the measurability conditions

$$
\begin{aligned}
& b(\cdot) x, x b(\cdot): M \rightarrow L^{2}(\mathscr{A}) \text { are strongly measurable } \quad \forall x \in\left(L^{2} \cap L^{\infty}\right)(\mathscr{A}) \\
& \|b(\cdot)\|_{\left(L^{2}+L^{\infty}\right)(\mathscr{A})} \text { is measurable. }
\end{aligned}
$$

Then $\left(Q_{b}, \mathscr{Q}_{b}\right)$ is a closed, completely Dirichlet form on $L^{2}(\mathscr{A})$ providing only that $\mathscr{Q}_{b}$ is $L^{2}$-norm dense.

Proof. Let $\mathscr{B}=\mathscr{A} \otimes L^{\infty}(M)$. First notice that if $M$ is finite and $b$ is essentially bounded, in the sense that there is $K<\infty$ such that $\|b(m)\|_{\left(L^{\infty}+L^{2}\right)(\mathscr{A})}<K$ for almost all $m$, then $b \in\left(L^{\infty}+L^{2}\right)(\mathscr{B})$ and $Q_{b}$ is the restriction of the closed, completely Dirichlet form $f \mapsto\left\|\bar{\delta}_{b} f\right\|_{L^{2}(\mathscr{B})}^{2}$ to constant functions $f . Q_{b}$ is thus closed and completely Dirichlet (cf. [DL] Proposition 6.11). For $\sigma$-finite $M$ and unbounded $b$ let $\left(M_{N}\right)$ be a sequence of finite measure subsets increasing to $M$, and let $b_{N}$ be $b$ times the indicator function of $M_{N} \cap\left\{m:\|b(m)\|_{\left(L^{\infty}+L^{2}\right)(\mathscr{A})} \leqq N\right\}$. Then $Q_{b}=\sup _{N} Q_{b_{N}}$, and so is closed and completely Dirichlet.

The generator defined rigorously by (4.5) has formal expression

$$
H x=B x+x B-2 \int_{M} b(m) x^{\Gamma} b(m) d m,
$$

where $B=\int b(m)^{2} d m$. Such generators were previously considered in connection with neutron diffusion equations ([D1, D2]).

Acknowledgement. We are grateful for support from the Science and Engineering Research Council, U.K., under grant number GR/E 68327.

\section{References}

[AH-K] Albeverio, S., Høegh-Krohn, R.: Dirichlet forms and Markov semigroups on $C^{*}$ algebras. Commun. Math. Phys. 56, 173-187 (1977)

[ApH] Applebaum, D.B., Hudson, R.L.: Fermion Itô's formula and stochastic evolutions. Commun. Math. Phys. 96, 473-496 (1984)

[ArY] Araki, H., Yamagami, S.: An inequality for Hilbert-Schmidt norm. Commun. Math. Phys. 81, 89-96 (1981)

[D1] Davies, E.B.: First and second quantised neutron diffusion equations. Commun. Math. Phys. 52, 111-126 (1977)

[D2] Davies, E.B.: Irreversible dynamics of infinite fermion systems. Commun. Math. Phys. 55, 231-258 (1977) 
[DL」 Davies, E.B., Lindsay, J.M.: Non-commutative symmetric Markov semigroups. Math. Zeit. 210, 379-411 (1992)

[Dix] Dixmier, J.: Von Neumann algebras (2nd ed., transl. F. Jellett). Amsterdam: NorthHolland 1981

[Ev, D] Evans, D.E.: Quantum dynamical semigroups, symmetry groups, and locality. Acta. Applic. Math. 2, 333-352 (1984)

[Ev, M] Evans, M.P.: Existence of quantum diffusions. Prob. Th. Rel. Fields 81, 473-483 (1989)

[Fuk] Fukushima, M.: Dirichlet forms and Markov processes. Amsterdam: North-Holland 1980

[GL1] Goldstein, S., Lindsay, J.M.: Beurling-Deny conditions for quantum dynamical semigroups. Preprint

[GL2] Goldstein, S., Lindsay, J.M.: KMS-symmetric Markov semigroups. Preprint

[Haa] Haagerup, U.: $L^{p}$-spaces associated with an arbitrary von Neumann algebra. In: Algebres d'operateurs et leur application en physique mathematique. Colloques internationaux du CNRS, no. 274, Marseilles 20-24 juin 1977, pp 175-184, Edition du CNRS, Paris 1979

[HuP] Hudson, R.L., Parthasarathy, K.R.: Quantum Itô's formula and stochastic evolutions. Commun. Math. Phys. 93, 301-323 (1984)

[Kun] Kunze, R.A.: $L^{p}$ Fourier transforms on locally compact unimodular groups. Trans. Am. Math. Soc. 89, 519-540 (1958)

[Nel] Nelson, E.: Notes on non-commutative integration. J. Funct. Anal. 15, 103-116 (1974)

[Par] Parthasarathy, K.R.: An Introduction to Quantum Stochastic Calculus. Basel, Boston: Birkhäuser 1992

[Seg] Segal, I.E.: A non-commutative extension of abstract integration. Ann. Math. 57, 401-456 (1953); Correction, Ann. Math. 58, 595-596 (1953)

[Sti] Stinespring, W.F.: Integration theorems for gages and duality for unimodular groups. Trans. Am. Math. Soc. 90, 15-56 (1959)

[Yea] Yeadon, F.J.: Non-commutative $L^{p}$-spaces. Math. Proc. Camb. Phil. Soc. 77, 91-102 (1975)

Communicated by H. Araki 